\title{
Spin currents and filtering behavior in zigzag graphene nanoribbons with adsorbed molybdenum chains
}

\author{
A García-Fuente ${ }^{1,2}$, L J Gallego ${ }^{3}$ and A Vega ${ }^{2}$ \\ ${ }^{1}$ Department of Chemistry, University of Fribourg, CH-1700 Fribourg, Switzerland \\ ${ }^{2}$ Departamento de Física Teórica, Atómica y Óptica, Universidad de Valladolid, E-47011 Valladolid, \\ Spain \\ ${ }^{3}$ Departamento de Física de la Materia Condensada, Facultad de Física, Universidad de Santiago de \\ Compostela, E-15782 Santiago de Compostela, Spain
}

E-mail: avega@fta.uva.es

\begin{abstract}
By means of density-functional-theoretic calculations, we investigated the structural, electronic and transport properties of hydrogen-passivated zigzag graphene nanoribbons (ZGNRs) on which a one-atom-thick Mo chain was adsorbed (with or without one or two missing atoms), or in which the passivating hydrogen atoms were replaced by Mo atoms. Mo-passivated ZGNRs proved to be nonmagnetic. ZGNRs with an adsorbed defect-free Mo chain were most stable with the Mo atoms forming dimers above edge bay sites, which suppressed the magnetic moments of the $\mathrm{C}$ atoms in that half of the ribbon; around the Fermi level of these systems, each spin component had a transmission channel via the Mo $s p_{z}$ band and one had an additional channel created by polarization of the ZGNR $\pi^{*}$ band, leading to a net spin current. The absence of an Mo dimer from an Mo chain adsorbed at the ZGNR edge made the system a perfect spin filter at low voltage bias by suppressing the Mo $s p_{z}$ band channels. Thus this last kind of hybrid system is a potential spin valve.
\end{abstract}

Keywords: graphene, transport properties, spintronics, DFT methods, transition metals

(Some figures may appear in colour only in the online journal)

\section{Introduction}

Graphene nanoribbons (GNRs) are quasi-one-dimensional derivatives of the graphene sheet that have attracted much attention in recent years because of their many potential applications in electronics and spintronics (see, e.g., [1-3] and references cited therein). Of particular interest are hydrogen-passivated zigzag GNRs (HPZGNRs), which have spin-polarized ground states in which the spin moments of the $\mathrm{C}$ atoms at each edge couple ferromagnetically to each other, while those of opposite edges are oriented antiferromagnetically. In this Ferro-A configuration all the $\mathrm{C}$ atoms couple antiferromagnetically to their nearest neighbours, whereas in the slightly less stable Ferro$\mathrm{F}$ configuration, in which the spin moments of the two edges are aligned ferromagnetically, between-neighbor antiferromagnetic coupling is frustrated at the nanoribbon centre (as a result of which the magnetic moments of these central atoms are almost completely suppressed) [4-7]. FerroA HPZGNRs are semiconductors, while Ferro-F HPZGNRs are metallic with one transmission channel for each spin component.

For spintronic applications it is necessary to modify the electronic structure of pristine HPZGNRs so that the charge transport properties of the two spins become different. This has been achieved in several ways, including the application of external electric fields [8], the replacement of edge $\mathrm{C}$ atoms by atoms of nonmagnetic species such as $\mathrm{N}$ and $\mathrm{B}$ (which inject electrons and holes, respectively) [5,9] and the adsorption of single atoms of magnetic transition metals (TMs) at the edge of the ribbon $[7,10]$.

It has recently been suggested that spin-polarized electronic currents can be sustained by nanostructures composed of zigzag GNRs (ZGNRs) and one-atom-thick linear 
chains of magnetic TM atoms that lie parallel to the direction of the ribbon (TMCs) [11-13]. Specifically, in a theoretical study of ZGNRs in which the edge carbons were all bound to Fe atoms and these to each other, Ong et al [11] found that around the Fermi level of the FM configuration (in which the magnetic moments of the Fe borders were aligned ferromagnetically) almost all density of states were of the same (minority) spin; and since the difference in energy between the FM configuration and the ground-state AFM configuration (in which the borders were aligned antiferromagnetically) was small enough to be easily overcome by an external magnetic field, the possibility of spintronic applications was suggested. However, calculations of the transport properties of this system using the non-equilibrium Green's function (NEGF) in conjunction with density-functional theory (DFT) have predicted that the two spins behave similarly [14].

In the above-mentioned studies of Ong et al [11] and Nguyen et al [14], the ZGNR-bound Fe TMCs evolved to form a chain of Fe dimers arranged end-to-end. ZGNR-bound molybdenum TMCs may be expected to exhibit this behaviour even more markedly because, due to its six half-filled valence orbitals, Mo forms homodimers with very strong $d-d$ bonds and an exceptionally short bond length (1.93 $\AA$ ) [15]. Indeed, in computational studies Mo clusters exhibit a strong tendency to exist as clusters of dimers [16] and infinite linear Mo chains as chains of dimers [17].

In this work we investigated the spintronic potential of ZGNR-bound Mo TMCs (Mo-passivated ZGNRs, MoPZGNRs); HPZGNRs with an Mo TMC adsorbed parallel to the midline (all possible midline-TMC distances were considered); and, in view of the results obtained for the preceding systems, HPZGNRs on which an Mo TMC lacking an Mo atom or two adjacent Mo atoms was adsorbed over edge bay sites. The structures and electronic and electron transport properties of these systems were calculated using DFT and the NEGF formalism. The computational methods used are briefly described in section 2; our findings are presented and discussed in section 3; and in section 4 we summarize our main conclusions.

\section{Computational methods}

Our calculations of structures and electronic properties were performed using the DFT package SIESTA [18] with the Perdew-Burke-Ernzerhof form of the generalized gradient approximation (GGA) [19]. We replaced the atomic cores by nonlocal norm-conserving Troullier-Martins pseudopotentials [20], which were factorized in the Kleinman-Bylander form [21] and generated using the atomic configuration $4 d^{5} 5 s^{1}$ $5 p^{0}$, with cutoff radii of $1.67,2.30$ and 2.46 a.u., respectively, in the case of Mo and $2 s^{2} 2 p^{2}$, with cutoff radii of 1.25 a.u., for C. For Mo, we also included nonlinear core corrections, generated with a radius of 1.2 a.u., to account for the overlap of the core charge with the valence $d$ orbitals and to avoid the spikes which often appear close to the nucleus when the GGA approximation is used. SIESTA employs a linear combination of pseudo-atomic orbitals to describe valence states. The basis set for Mo included double- $\zeta$ polarized orbitals, i.e. two radial

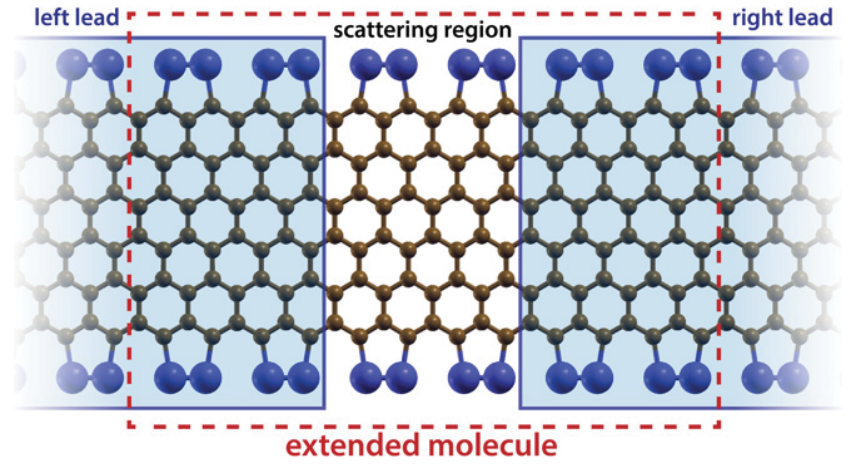

Figure 1. Schematic representation of the model used in the calculations (the case shown is 6-MoPZGNR). The 'extended molecule' comprises the scattering region together with electrodes that are short proximal segments of leads formed by semi-infinite nanoribbons. The whole infinite structure is periodic except for the calculations reported in section 3.3 , for which defects were introduced in a larger scattering region (see section 3.3 for details).

functions to describe the $5 s$ shell and another two for each $d$ state of the $4 d$ shell, plus a single radial function for each $p$ state of the empty $p$ shell. For $\mathrm{C}$, we used a double- $\zeta$ basis with two radial functions to describe the $2 s$ shell and another two for each $p$ state of the $2 p$ shell. A double- $\zeta$ basis was also used for the passivating $\mathrm{H}$ atoms. SIESTA uses a numerical grid to compute the exchange and correlation potential and to perform the real-space integrals that yield the Hamiltonian and overlap matrix elements. We defined this grid with an energy cutoff of $250 \mathrm{Ry}$ and used $10 k$ points in the $z$ direction (the direction of growth of the GNR), which was found to be sufficient for convergence of energies and band structures. In calculating the density matrix we smoothed the Fermi distribution function with an electronic temperature of $10 \mathrm{meV}$ and we used a conjugate gradient method [22] to relax atomic positions until interatomic forces were smaller than $0.005 \mathrm{eV} \AA^{-1}$.

Conductance in the linear regime was computed with SMEAGOL [23], a flexible and efficient implementation of the NEGF formalism that is specially designed for calculation of the transport properties of nanoscale systems. In the present work, SMEAGOL applies the SIESTA framework (basis orbitals, pseudopotentials, etc) to the Hamiltonian for an open system comprising a finite segment of the nanoribbon (the 'extended molecule') that consists of three parts: a central part (treated as a scattering region) plus, on either side, a short proximal segment of a semi-infinite lead (a 'contact region'). SMEAGOL calculates electronic density and transmission using the NEGF formalism [24]. Since the low-voltage differential conductance $G(V)=\mathrm{d} I / \mathrm{d} V$ can be approximated by

$G(V) \simeq \sum_{\sigma} G_{0}\left(T_{\sigma}(0,0)+2 \frac{\partial T_{\sigma}}{\partial V} V\right) \simeq \sum_{\sigma} G_{0} T_{\sigma}(0,0)$

(where $G_{0}=e^{2} / h$ is the quantum of conductance for magnetic systems and $T_{\sigma}(0,0)$ is the zero-voltage transmission for the spin component $\sigma$ at the Fermi level $E_{\mathrm{F}}$, which is taken as the energy origin), the zero-voltage transmission at $E_{\mathrm{F}}$ provides an estimate of the differential conductance in the linear regime. 


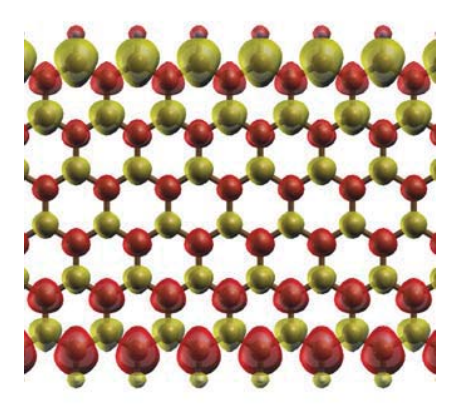

6-HPZGNR Ferro-A

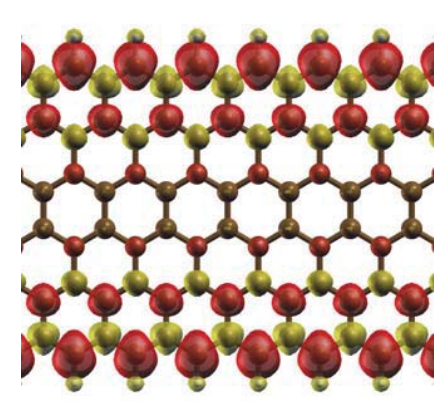

6-HPZGNR Ferro-F

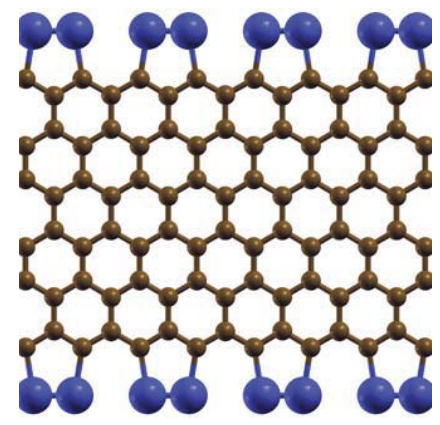

6-MoPZGNR

Figure 2. Spin density distributions of Ferro-A 6-HPZGNR, Ferro-F 6-HPZGNR and 6-MoPZGNR. Red (dark shading) indicates positive values (net spin up), yellow (light shading) negative values (net spin down).
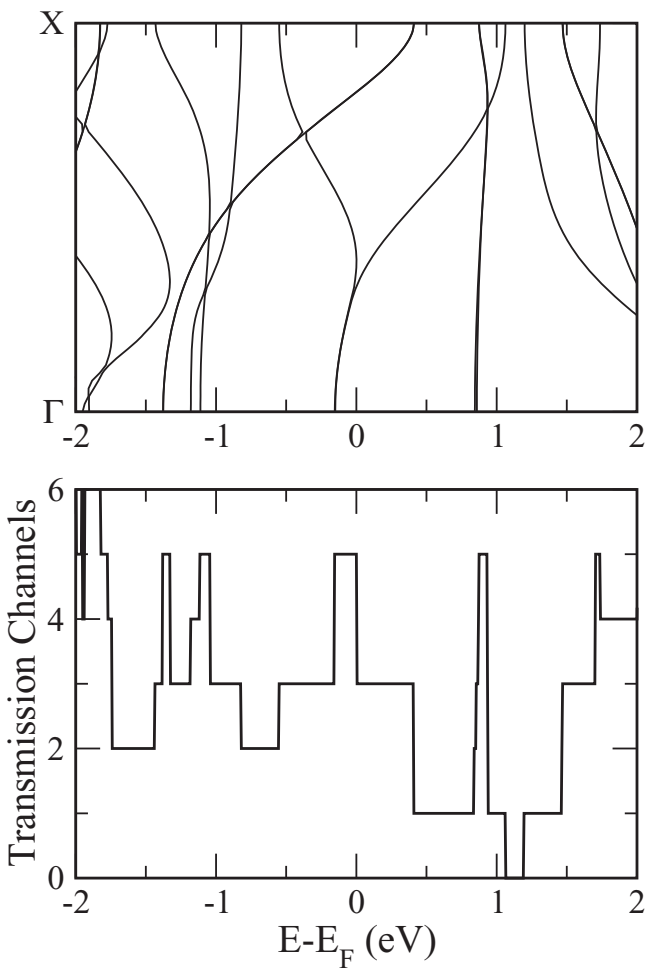

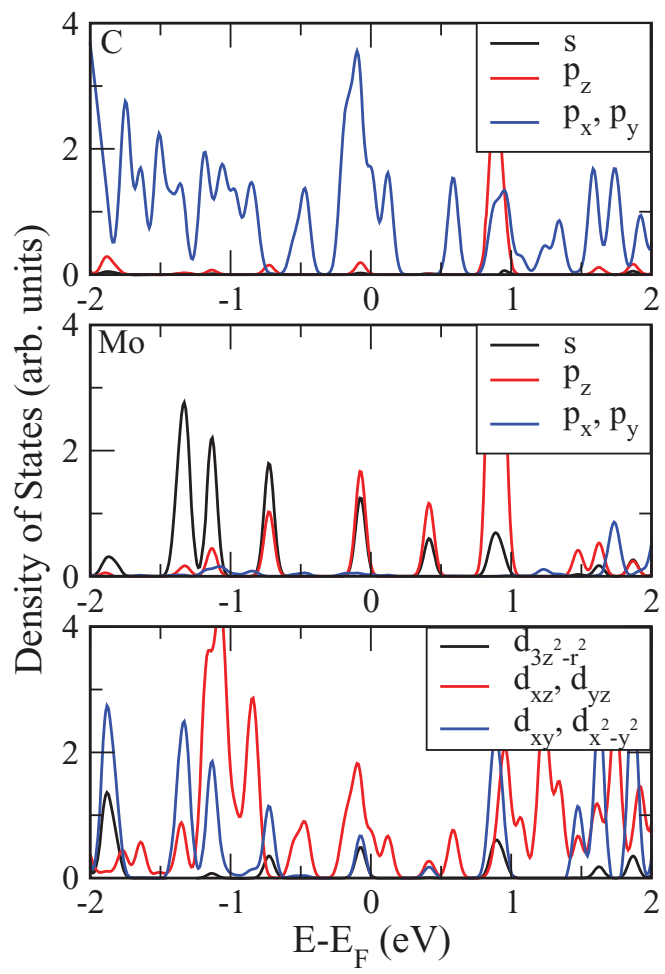

Figure 3. Band structures, transmission channels and densities of states projected on the pseudo-atomic orbitals of the model, for either spin component of 6-MoPZGNR.

When we modeled systems with defective TMCs lacking Mo atoms, these defects were located in the scattering region; when the TMCs had no defects, the scattering region was no different from the leads. Note that in this latter situation (a onedimensional periodic system) transport can be considered as ballistic (i.e. there is no scattering) and the zero-voltage transmission coefficient for a given energy just counts the number of bands of this energy, i.e. the number of transmission channels.

\section{Results and discussion}

\subsection{Mo-passivated ribbons}

We first investigated Mo-passivated ZGNRs, i.e. ZGNRs with Mo atoms instead of $\mathrm{H}$ atoms bound to the $\mathrm{C}$ atoms at the edges (we use the notations ' $N$-MoPZGNRs' and ' $N$-HPZGNRs' to refer respectively to Mo- and H-passivated ZGNRs composed of $N$ zigzag $\mathrm{C}$ chains in the direction in which the ribbon progresses). $N$ ranged from 5 to 10 and both Ferro-A and Ferro-F configurations were considered. The calculations were performed for extended molecules consisting of six supercells, each comprising two unit cells of the ZGNR and four Mo atoms, two on each edge. The central two supercells were treated as the scattering region and the other four as its flanking contact (electrode) regions (see figure 1). Calculations were also performed for pristine $N$-HPZGNR systems for comparison.

The Mo binding energy,

$$
E_{\mathrm{B}}(\mathrm{Mo})=\frac{E(\mathrm{ZGNR})+4 E(\mathrm{Mo})-E(\mathrm{MoPZGNR})}{4},
$$

was for all ribbon widths $4.33 \mathrm{eV}$, a value somewhat smaller than the $4.87 \mathrm{eV}$ of the $\mathrm{H}$ atoms of HPZGNR but larger 


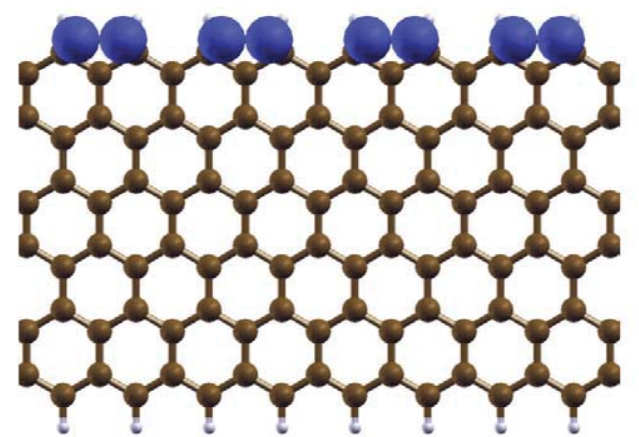

Mo(a)@6-HPZGNR

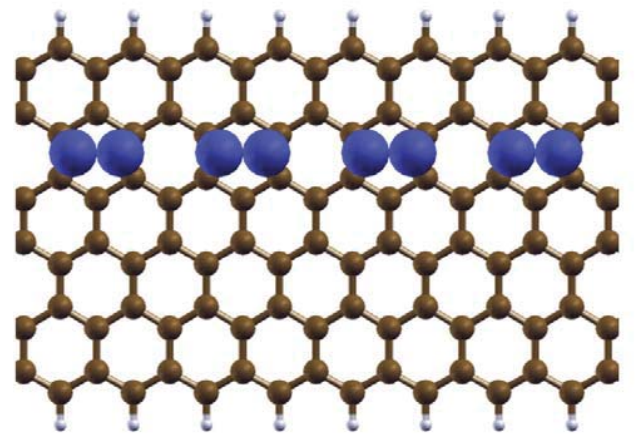

Mo(c)@6-HPZGNR

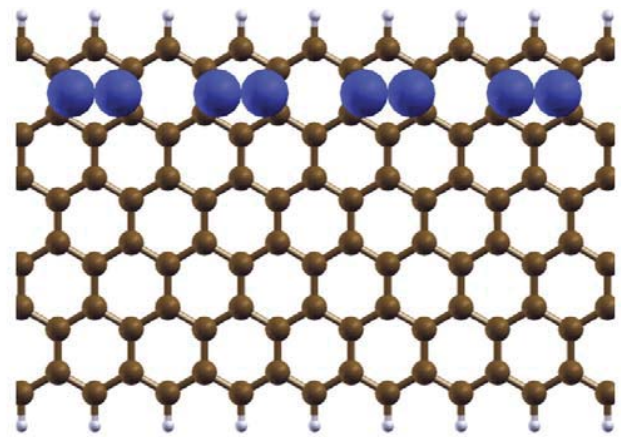

Mo(b)@6-HPZGNR

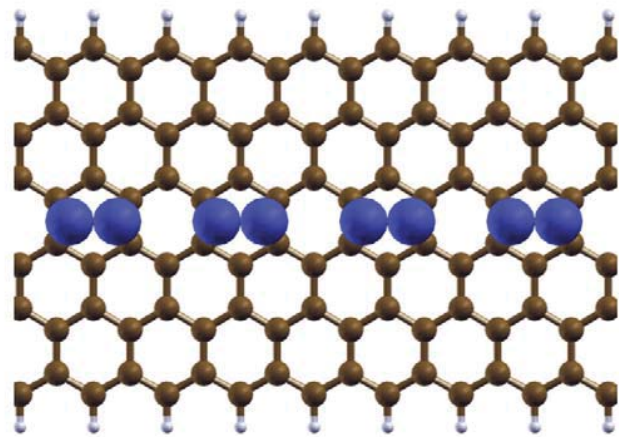

Mo(d)@6-HPZGNR

Figure 4. Stable structures of Mo@6-HPZGNR. Stability decreases from Mo(a)@6-HPZGNR to Mo(d)@6-HPZGNR following the alphabetic order of the infix.
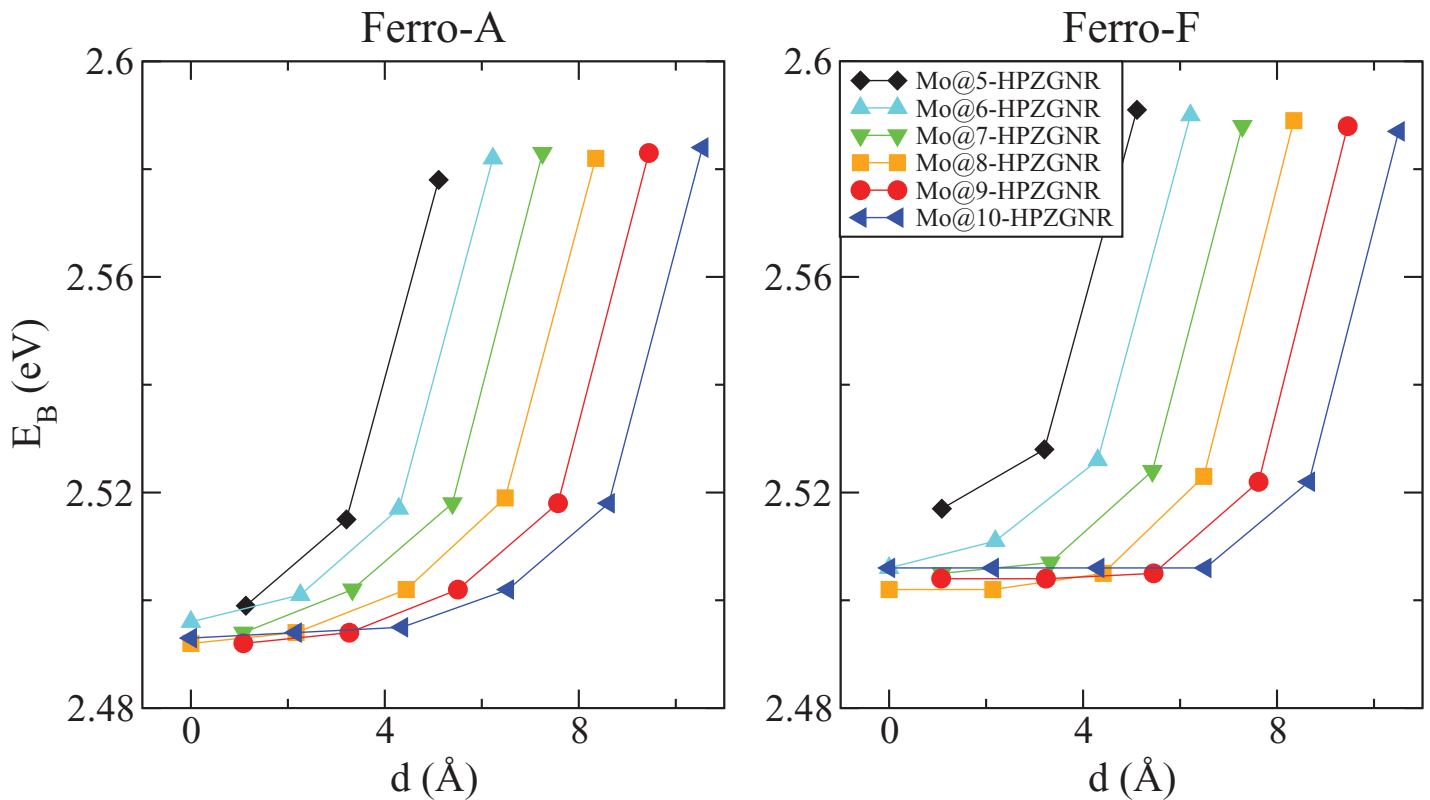

Figure 5. Binding energies $E_{\mathrm{B}}$ of the Mo atoms in the Ferro-A and Ferro-F configurations of Mo@ $N$-HPZGNRs, plotted against the distance $d$ of the Mo chain from the center of the ribbon.

than the $4.23 \mathrm{eV}$ of $\mathrm{Fe}$ atoms in analogous FePZGNRs [11]. Like the Fe passivators of these latter systems, the Mo atoms formed dimers. However, unlike pristine HPZGNRs [4-7] and FePZGNRs [11, 14], MoPZGNRs are not magnetic.
By way of illustration, figure 2 compares the spin density distribution of 6-MoPZGNR with those we obtained for the Ferro-A and Ferro-F configurations of 6-HPZGNR and figure 3 shows the electronic band structure, transmission channels and 


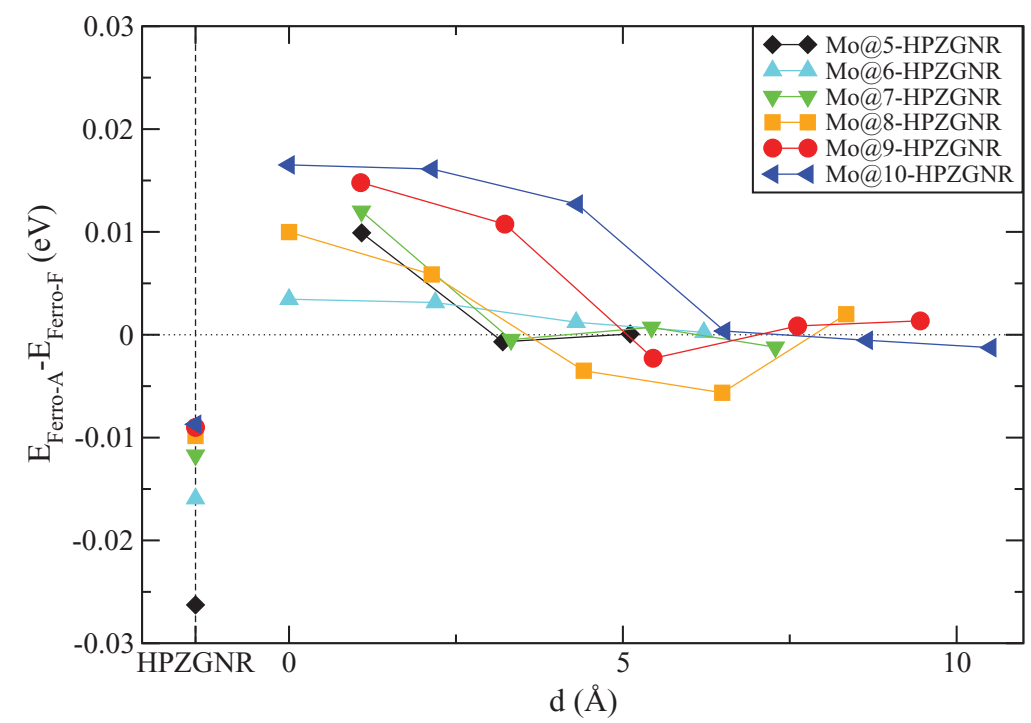

Figure 6. Energy differences between the Ferro-A and Ferro-F configurations of Mo@ $N$-HPZGNRs, plotted against the distance $d$ of the Mo chain from the center of the ribbon, together with the corresponding differences for the pristine HPZGNRs (points on the vertical dashed line).

densities of states of 6-MoPZGNR. At the Fermi level there are three transmission channels for each spin, two provided by a degenerate band extending from -1.4 to $0.4 \mathrm{eV}$ and another by a band that extends from -0.2 to $1.1 \mathrm{eV}$. Although the extensive hybridization makes it difficult to assign bands to particular states, these channels clearly receive significant contributions from the Mo $d$ states. The fact that the two spins have the same number of channels rules out the spintronic use of $N$-MoPZGNRs.

\subsection{Adsorbed defect-free Mo chains}

We next investigated Mo@ $N$-HPZGNRs, i.e. systems comprising a single Mo TMC adsorbed on an $N$-HPZGNR. The extended molecules of the model again consisted of six supercells (two for the scattering region and two for each contact region), but each supercell now comprised two unit cells of the HPZGNR and two Mo atoms. The initial positions of the latter were always such as to form an infinite linear chain, but varied in the separation of the two atoms, their distance from the centre of the nanoribbon and the position of the leading atom in the direction of the nanoribbon.

By way of illustration, figure 4 shows the stable configurations of Ferro-A Mo@6-HPZGNR. As in the other Mo@N-HPZGNR systems, all exhibit strong Mo dimerization, each dimer lying horizontally over one hole or edge bay of the carbon lattice, parallel to the direction of progression of the ribbon and separated from the next dimer by an unoccupied hole or bay. Stability increases with the distance $d$ of the TMC from the center of the HPZGNR; figure 5 shows, for all the systems investigated, the $d$-dependence of the Mo binding energy, defined as

$E_{\mathrm{B}}=\frac{E(\mathrm{HPZGNR})+2 E(\mathrm{Mo})-E(\mathrm{HPZGNR}+2 \mathrm{Mo})}{2}$,

where $E(\mathrm{HPZGNR})$ is the energy of the pristine nanoribbon in the magnetic configuration (Ferro-A or Ferro-F) that obtains at the start of the simulation leading to $E(\mathrm{HPZGNR}+2 \mathrm{Mo})$. The behavior displayed in this figure allows one to distinguish between a central zone around the midline of the ribbon, in which the Mo binding energy varies very little with the position of the Mo chain, and lateral zones embracing the two or three binding positions farthest from the center, where the binding energy increases steeply as the edge is approached. The increase in stability with increasing $d$ is accompanied by a decrease in the height of the Mo atoms above the ribbon, which falls from $3.3-3.4 \AA$ in the central zone to about $2.9 \AA$ above edge bays.

Figure 5 also shows that in all cases the Mo binding energy is greater when the unrelaxed nanoribbon was Ferro- $F$ than when it was Ferro-A, especially for small $N$ or when the TMC is adsorbed in the central zone, where the difference reaches about $0.01 \mathrm{eV}$ and inverts the relative energies of the Ferro$A$ and Ferro-F forms (figure 6). However, the mechanism by which Mo binding stabilizes the Ferro-F configuration is not clear. Certainly, it does not seem to consist in straightforward local magnetic stabilization of ferromagnetic nearest-neighbor $\mathrm{C}-\mathrm{C}$ interactions where antiferromagnetic coupling is frustrated, because $(a)$ the magnetic moment of the Mo atoms is small or zero in the central zone (figure 7), (b) the frustration is in many cases not located at the TMC adsorption site (see below) and $(c)$ adsorption of the TMC at the edge converts an initial Ferro-F configuration into a 'hemi-Ferro-A' form in which all $\mathrm{C}$ atoms couple antiferromagnetically to their nearest neighbors (see below).

All $\mathrm{C}$ atoms between the TMC and the midline have magnetic moments less than $0.010-0.015 \mu_{\mathrm{B}}$, the largest value decreasing with increasing $N$. With the exceptions described in the next paragraph, there is also some depression of moments closer to the near edge, while the moments of $\mathrm{C}$ atoms between the midline and the far edge are little affected by the adsorption of the TMC (figure 7). Although in most cases Mo chains at the very centre of the ribbon, or immediately adjacent to the 
Ferro-A
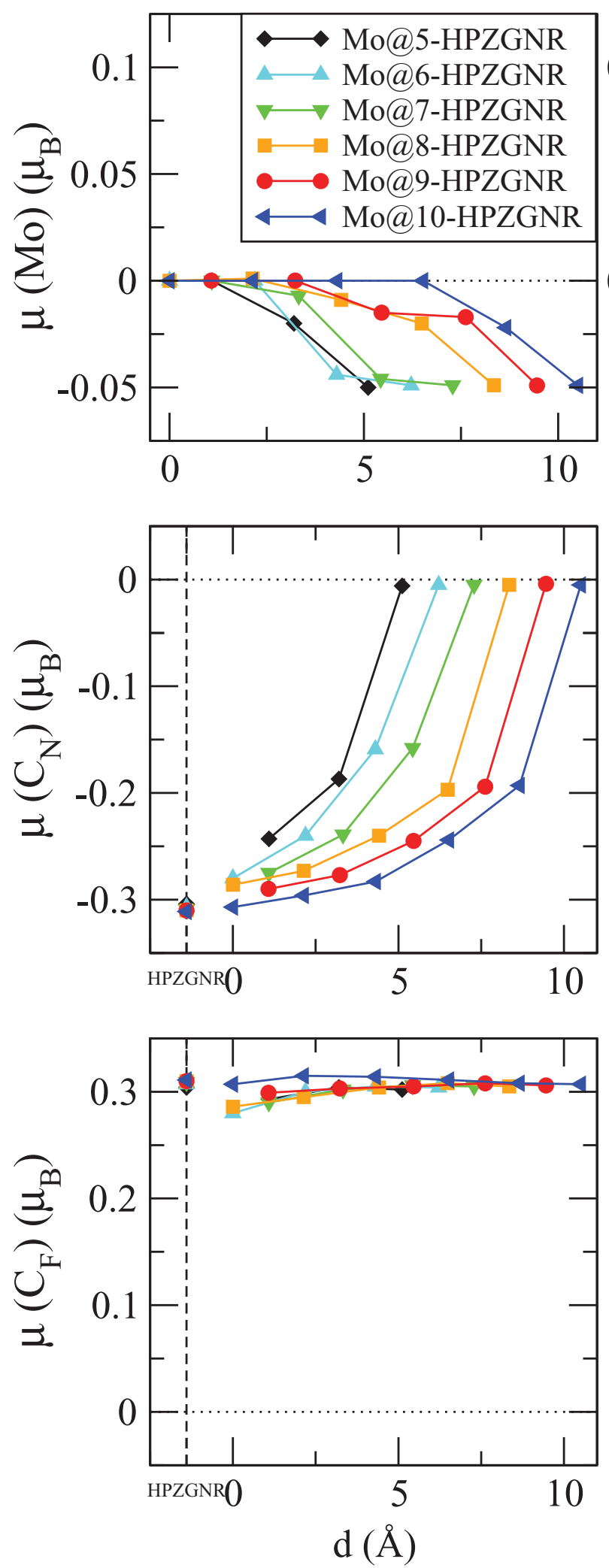

Ferro-F
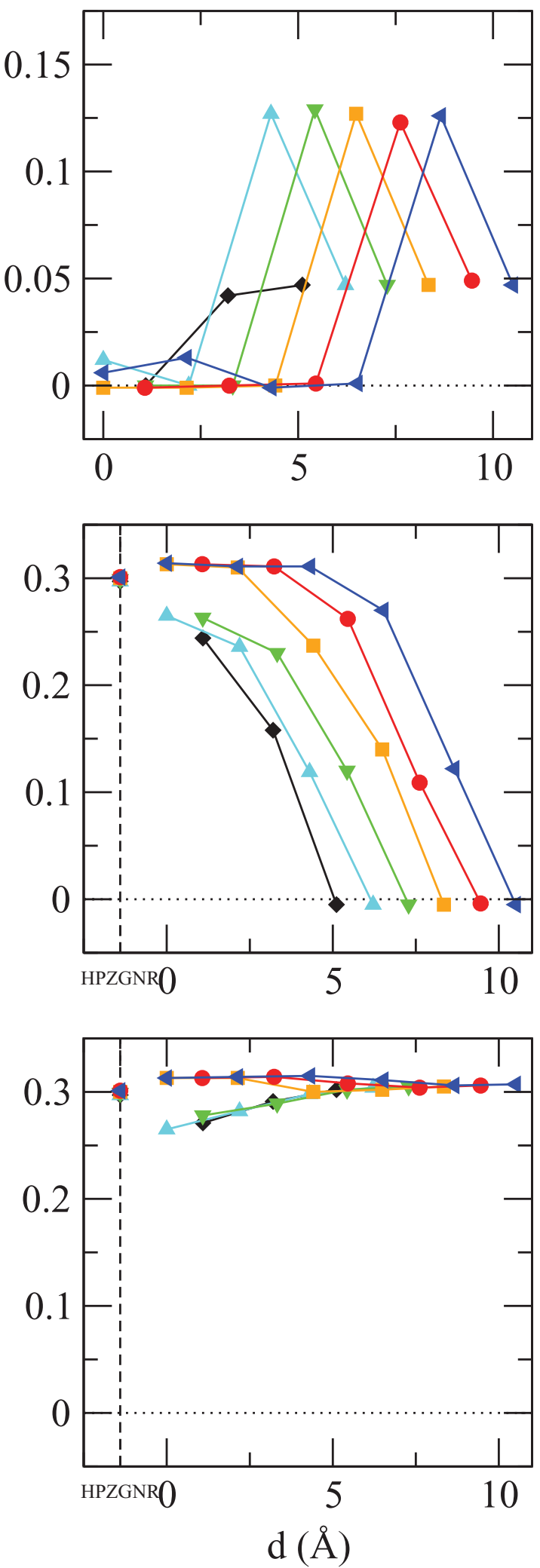

Figure 7. Magnetic moments of Mo atoms, near-edge $\mathrm{C}$ atoms $\left(\mathrm{C}_{N}\right.$, i.e. $\mathrm{C}$ atoms at the edge of the nanoribbon nearer the Mo chain) and far-edge $\mathrm{C}$ atoms $\left(\mathrm{C}_{\mathrm{F}}\right)$, in the Ferro-A and Ferro-F configurations of Mo@ $N$-HPZGNRs, plotted against the distance $d$ of the Mo chain from the center of the ribbon. The points on the vertical dashed lines show the magnetic moments of the $\mathrm{C}$ atoms of the two edges in the pristine HPZGNRs. 
Ferro-A

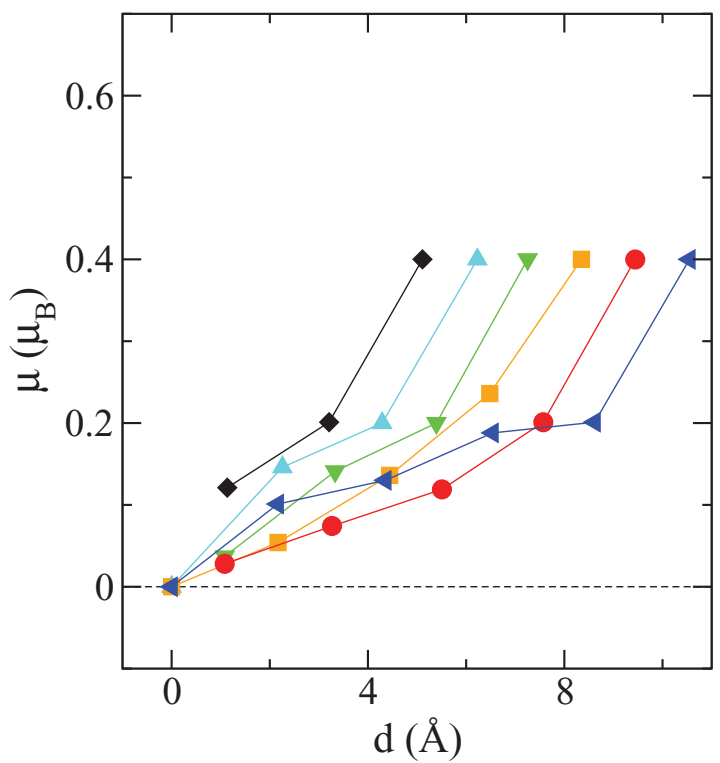

\section{Ferro-F}

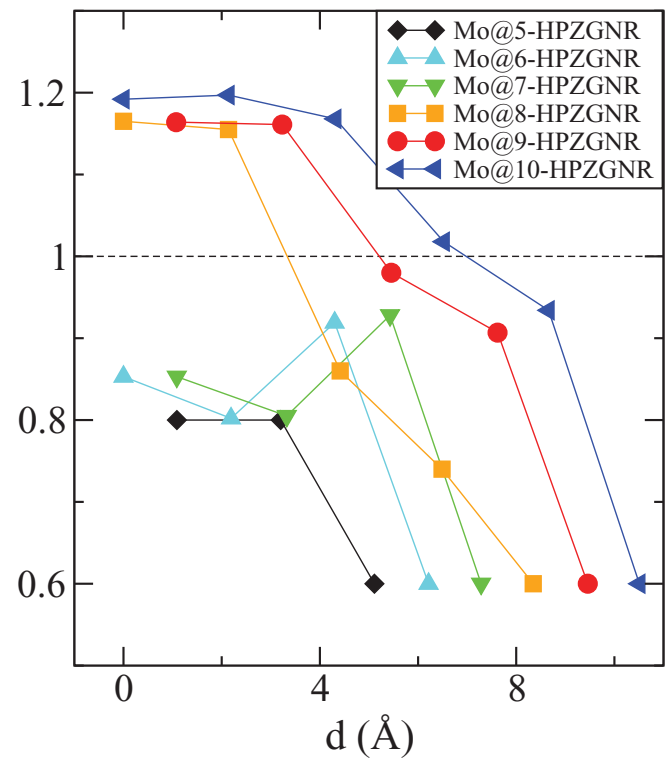

Figure 8. Total magnetic moment per unit cell of the Ferro-A and Ferro-F configurations of Mo@ $N$-HPZGNRs plotted against the distance $d$ of the Mo chain from the center of the ribbon. The dashed lines indicate the corresponding values of pristine HPZGNRs.
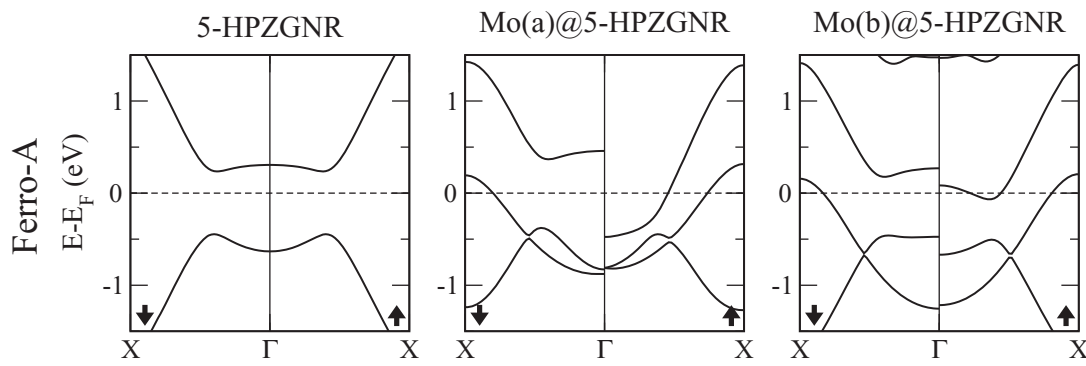

Mo(c)@5-HPZGNR
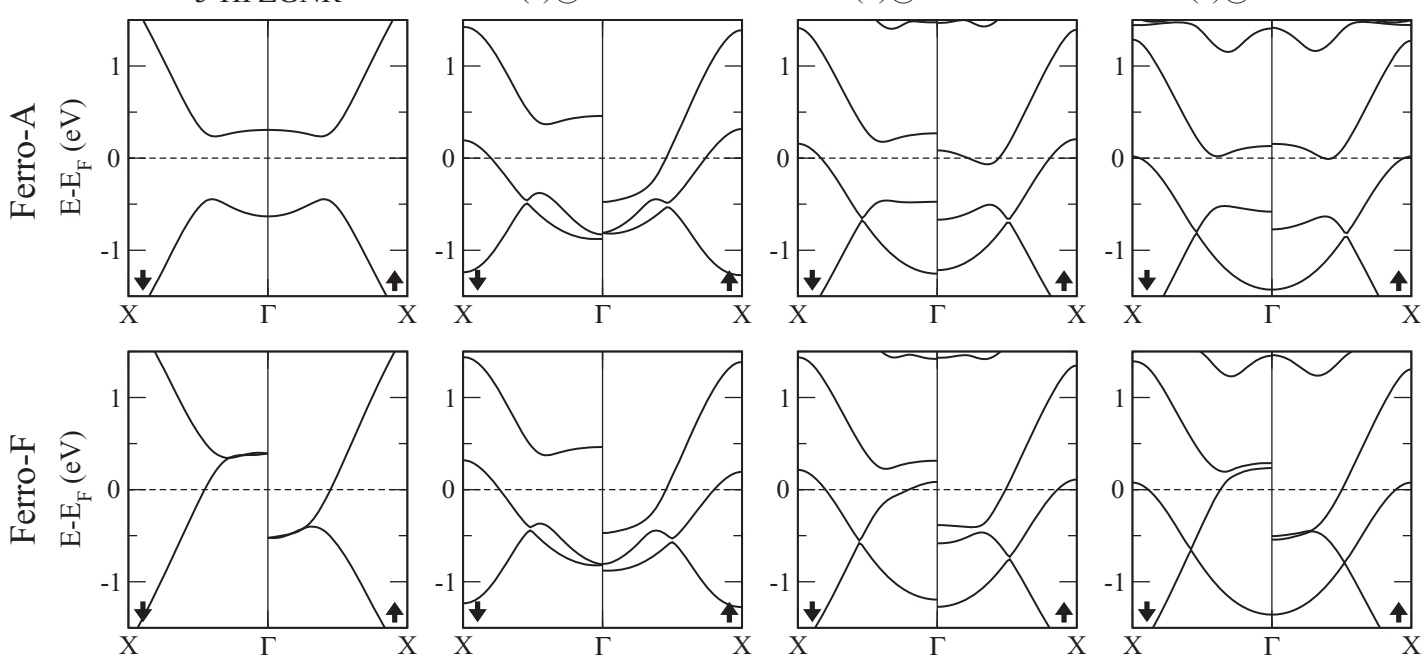

Figure 9. Band structures of 5-HPZGNR and of $\operatorname{Mo}(x) @ 5$-HPZGNR systems, where, as in figure 4, $x=a$ when the Mo chain lies over the edge of the HPZGNR (the most stable configuration) and $\mathrm{c}$ when it lies at the center of the ribbon (the least stable configuration).

central $\mathrm{C}$ row if $N$ is odd, are flanked on either side by $\mathrm{C}$ atoms that are mutually ferromagnetic, the site of $\mathrm{C}-\mathrm{C}$ magnetic frustration tends to trail behind the TMC chain as it crosses the central zone and then catch up with it as it crosses the lateral zone. When the TMC binds over edge bays, all $\mathrm{C}$ atoms couple antiferromagnetically to their nearest neighbors, the magnetic moments of $\mathrm{C}$ atoms in the near half of the ribbon are very small (while those of the other half are virtually the same as in a pristine HPZGNR) and the Mo atoms, which have acquired magnetic moments of around $0.05 \mu_{\mathrm{B}}$ due to polarization of their $s p_{z}$ states, couple ferromagnetically to the near-edge $\mathrm{C}$ atoms of ribbons that were initially Ferro-A and in general antiferromagnetically to those of ribbons that were initially Ferro-F. We shall continue to use 'Ferro-A' and 'Ferro-F' to distinguish between these two configurations with edge-bound Mo chains, but these terms should in this context now be understood as indicating the relative orientations of the magnetic moments of Mo atoms and far-side edge carbons, not the relative orientations of the carbons at the two edges.

The exceptions referred to above mainly concern the broader Ferro-F ribbons $(N=8-10)$. In these systems, carbon magnetic moments are slightly greater than in the pristine HPZGNR both at the far edge and, when the TMC binds in the central zone, at the near edge; and to a lesser extent the same is true of Ferro-A Mo@10-HPZGNR, except that in this case the increase in near-edge moments only occurs when the TMC binds at the midline (figure 7). A further marked difference between the Ferro-A and Ferro-F series is that whereas on Ferro-A ribbons the Mo atoms acquire progressively greater magnetic moments as they cross the lateral zone, on Ferro-F 
Table 1. Transmission channels for each spin at the Fermi level in Mo@ $N$-HPZGNR systems $(N=5-10$; configurations are ordered by stability as in figure 4 ) and in the pristine HPZGNRs.

\begin{tabular}{llllll}
\hline & \multicolumn{2}{c}{ Ferro-A } & & Ferro-F \\
\cline { 2 - 3 } \cline { 5 - 5 } System & Spin up & Spin down & & Spin up & Spin down \\
\hline 5-HPZGNR & 0 & 0 & 1 & 1 \\
Mo(a)@5-HPZGNR & 2 & 1 & 2 & 1 \\
Mo(b)@5-HPZGNR & 3 & 1 & 2 & 2 \\
Mo(c)@5-HPZGNR & 3 & 1 & 2 & 2 \\
6-HPZGNR & 0 & 0 & 1 & 1 \\
Mo(a)@6-HPZGNR & 2 & 1 & 2 & 1 \\
Mo(b)@6-HPZGNR & 3 & 1 & 1 & 2 \\
Mo(c)@6-HPZGNR & 3 & 1 & 2 & 2 \\
Mo(d)@6-HPZGNR & 3 & 3 & 2 & 2 \\
7-HPZGNR & 0 & 0 & 1 & 1 \\
Mo(a)@7-HPZGNR & 2 & 1 & 2 & 1 \\
Mo(b)@7-HPZGNR & 3 & 1 & 1 & 2 \\
Mo(c)@7-HPZGNR & 3 & 1 & 2 & 2 \\
Mo(d)@7-HPZGNR & 3 & 3 & 2 & 2 \\
8-HPZGNR & 0 & 0 & 1 & 1 \\
Mo(a)@8-HPZGNR & 2 & 1 & 2 & 1 \\
Mo(b)@8-HPZGNR & 3 & 1 & 2 & 2 \\
Mo(c)@8-HPZGNR & 3 & 3 & 2 & 2 \\
Mo(d)@8-HPZGNR & 3 & 3 & 2 & 2 \\
Mo(e)@8-HPZGNR & 3 & 3 & 2 & 2 \\
9-HPZGNR & 0 & 0 & 1 & 1 \\
Mo(a)@9-HPZGNR & 2 & 1 & 2 & 1 \\
Mo(b)@9-HPZGNR & 3 & 1 & 2 & 1 \\
Mo(c)@9-HPZGNR & 3 & 3 & 2 & 2 \\
Mo(d)@9-HPZGNR & 3 & 3 & 2 & 2 \\
Mo(e)@9-HPZGNR & 3 & 3 & 2 & 2 \\
10-HPZGNR & 0 & 0 & 1 & 1 \\
Mo(a)@10-HPZGNR & 2 & 1 & 2 & 1 \\
Mo(b)@10-HPZGNR & 3 & 1 & 2 & 1 \\
Mo(c)@10-HPZGNR & 3 & 3 & 2 & 2 \\
Mo(d)@10-HPZGNR & 3 & 3 & 2 & 2 \\
Mo(e)@10-HPZGNR & 3 & 3 & 2 & 2 \\
Mo(f)@10-HPZGNR & 3 & 3 & 2 & 2 \\
\hline & & & 2 \\
\end{tabular}

ribbons their magnetic moment peaks at around $0.13 \mu_{\mathrm{B}}$ when they bind at next-to-edge sites, the only exception being FerroF Mo@5-HPZGNR (figure 7).

As a result of the above behavior, the total magnetic moment of a Ferro-A Mo@ $N$-HPZGNR per unit cell is very small when the Mo chain is adsorbed on its central zone (zero, as in the pristine ribbon, when $N$ is even and the Mo chain is right at the center of the ribbon); increases more or less linearly with the distance of the Mo chain from the center, until the last non-edge binding site is reached; and then jumps to $0.4 \mu_{\mathrm{B}}$ when the Mo chain lies over edge bays (figure 8). In this last configuration, the near half of the HPZGNR has near-zero total magnetic moment and the approximately $0.5 \mu_{\mathrm{B}}$ per unit cell of the far half (the same as in the pristine Ferro-A HPZGNR) has the opposite orientation to the $0.1 \mu_{\mathrm{B}}$ of the two Mo atoms. The total magnetic moment per unit cell of a Ferro-F Mo@NHPZGNR with its Mo chain at the midline is somewhat smaller than the $1 \mu_{\mathrm{B}}$ of the pristine HPZGNR if $N=5-7$, but larger if $N=8-10$ (figure 8). Total magnetic moment decreases as the Mo chain nears the edge, except that the high Mo moment at the next-to-edge site causes a peak in the total moment for $N=6$ and 7 . When the Mo chain is adsorbed over edge bays, the total magnetic moment is $0.6 \mu_{\mathrm{B}}$ per unit cell, $0.5 \mu_{\mathrm{B}}$ due to the far half of the HPZGNR and $0.1 \mu_{\mathrm{B}}$ to the Mo atoms.

Figure 9 shows the band structures of the Ferro-A and Ferro-F configurations of the Mo@5-HPZGNR systems together with those of pristine 5-HPZGNR for comparison; similar results were obtained for other values of $N$. In FerroA configuration, the Mo chain adsorbed at the center of the ribbon (Mo(c)@5-HPZGNR) creates a wide band between -1.4 and $0 \mathrm{eV}$ due to the Mo $s p_{z}$ states; lowers the energy of the $\pi^{*}$ band, which also becomes very slightly polarized; and likewise slightly polarizes the $\pi$ band. As the Mo chain moves toward the edge of the ribbon, the $\pi^{*}$ band becomes increasingly polarized until, in Mo(a)@5-HPZGNR, with the Mo chain at the edge, it is as strongly polarized as in the FerroF configuration of pristine 5-HPZGNR. The Mo $s p_{z}$ band is likewise slightly magnetized, though never by more than about $0.1 \mathrm{eV}$, while the polarization of the $\pi$ band disappears. In Ferro-F configuration, a central Mo chain introduces an $s p_{z}$ band as expected (now between -1.3 and $0.1 \mathrm{eV}$ ), but hardly affects the polarization of the $\pi$ and $\pi^{*}$ bands; and shifting the Mo chain to the edge of the ribbon once more slightly polarizes the $s p_{z}$ band (but in the opposite direction) and depolarizes the $\pi$ band while leaving the polarization of the $\pi^{*}$ band as in pristine Ferro-F 5-HPZGNR. When the Mo chain is at the edge, the band structures of systems that were initially Ferro-A and Ferro-F thus differ mainly in the orientation of the slight $s p_{z}$ polarization relative to the $\pi^{*}$ polarization, in keeping with the meagre difference in energy between these configurations (figure 6). In both cases the band structure of the ZGNR in Mo(a)@5-HPZGNR is similar to that of Ferro-A 5-HPZGNR for one spin component and similar to that of Ferro-F 5HPZGNR for the other spin component. This is because charge is transferred from the Mo chain to the ZGNR, partially filling the antibonding $\pi^{*}$ band of one spin component. A similar charge transfer has been observed in nanostructures consisting of Mo wires encapsulated in carbon nanotubes [25].

Table 1 lists, for all the Mo@N-HPZGNR systems and pristine $N$-HPZGNR, the predicted number of transmission channels for each spin at the Fermi level. Since there is very little hybridization between states around the Fermi level in these systems, each transmission channel can be associated with either the Mo chain or the nanoribbon. When the Mo chain is at the edge of the ribbon, i.e. in Mo(a)@N-HPZGNRs, spin-polarized currents are always possible, because both the Ferro-A and Ferro-F configurations feature two channels for the majority spin and just one for the minority spin, the Mo $s p_{z}$ states affording one channel for each spin and the nanoribbon a single spin-up channel (see figures 9 and 10). By contrast, when the Mo chain lies in the central zone of the ribbon the two spins always have the same number of transmission channels in Ferro-F configuration (one $s p_{z}$ channel and either a $\pi$ or a $\pi^{*}$ channel) and generally do in Ferro-A configuration (one $s p_{z}$ channel and two $\pi^{*}$ channels). The exceptional Ferro-A cases (Mo@5-HPZGNR and Mo@6- and -7-HPZGNR with Mo chains at the border of the central zone) are due to the slight polarization of the $\pi^{*}$ band, which results in the Fermi level being crossed by only one of its spin components. However, since the energy gap between the spin-up and spin-down $\pi^{*}$ 
Ferro-A

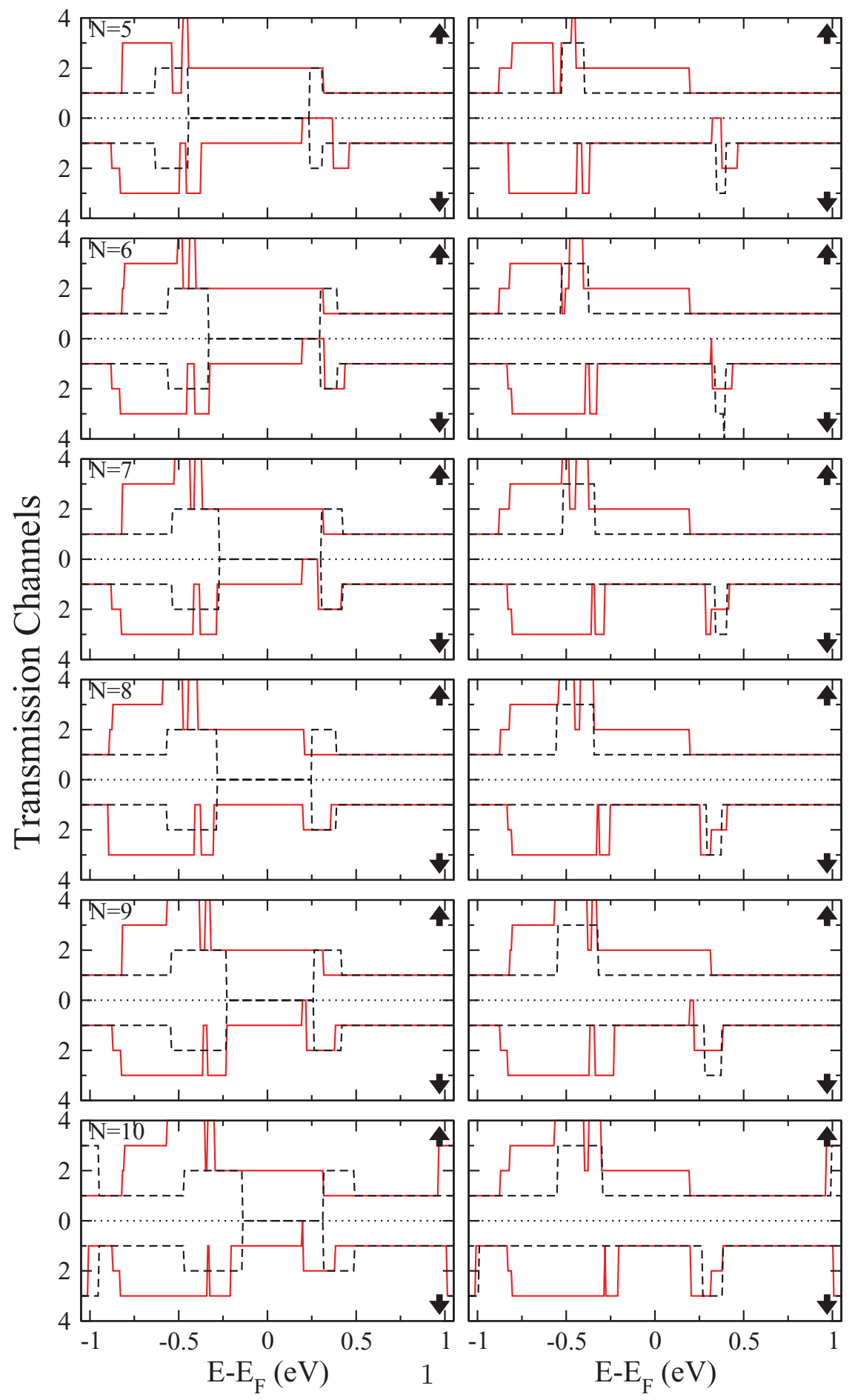

Figure 10. Spin-up ( $\uparrow)$ and spin-down $(\downarrow)$ electronic transmission channels of Ferro-A and Ferro-F Mo(a)@ $N$-HPZGNR systems (continuous red lines) and of the pristine $N$-HPZGNRs (pecked black lines), as functions of energy.

bands is very small, only very small bias voltages would produce measurable differences between spin-up and spindown currents.

\subsection{Influence of defects in the adsorbed Mo chain}

As noted above, in Mo(a)@N-HPZGNRs the Mo $s p_{z}$ states afford one transmission channel for each spin and the nanoribbon a single spin-up channel. Suppression of the Mo $s p_{z}$ channels would therefore result in a perfect spin filter. To investigate whether this might be achieved by introducing vacancies in the Mo chain, we computed the electronic transmittance of Ferro-A Mo(a)@6-HPZGNR systems with Mo chains lacking either a single atom or the two atoms of a single dimer. For this purpose we used an extended molecule comprising seven supercells of 

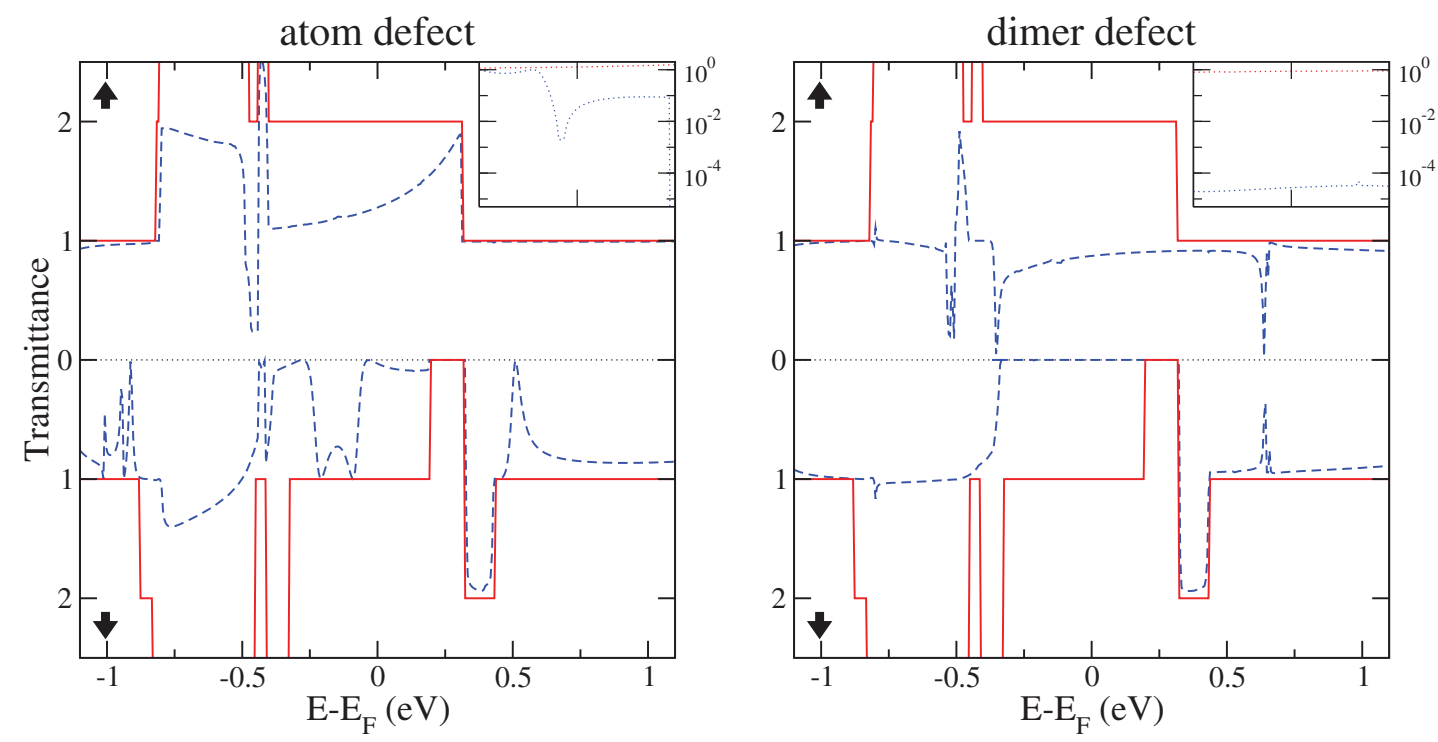

Figure 11. Dashed blue curves: spin-up $(\uparrow)$ and spin-down $(\downarrow)$ electronic transmittances of Ferro-A Mo(a)@ 6-HPZGNR systems lacking a single Mo atom (left panel) or an Mo dimer (right panel). For comparison, continuous red lines show the transmittances of periodic, defect-free Ferro-A Mo(a)@6-HPZGNR. Insets show the transmittances of the defect-bearing systems in the range $\left(E_{\mathrm{F}}-0.2 \mathrm{eV}\right.$,

$E_{\mathrm{F}}+0.2 \mathrm{eV}$ ) on a logarithmic scale, with dotted red curves for the spin-up component and dotted blue curves for the spin-down component.

Mo(a)@6-HPZGNR: a three-supercell scattering region in which the central supercell was modified by removal of one or both Mo atoms, flanked as before by two-supercell contact regions.

The calculated transmittances are presented in figure 11 and in figure 12 we show the corresponding spin densities together with spatial representations of the local densities of states (LDOS) of each spin component around the Fermi level; in both these figures the results for the defect-free system are also included for comparison. The top panel of figure 12 shows that, as was already deduced in the previous subsection from the band structure of the periodic defect-free system, this system can transmit spin-up current via both the Mo wire and the nanoribbon, but can only transmit spin-down current via the Mo wire. The other two panels of figure 12 show that the removal of Mo atoms from the Mo chain affects the density of states only very weakly on the nanoribbon, but very strongly on the Mo chain: LDOS on the Mo chain in the scattering region is significantly decreased by the removal of one Mo atom and practically eliminated by the removal of two, as a consequence of which transmission via the Mo $s p_{z}$ states is suppressed while spin-up transmission via the nanoribbon is preserved (figure 11). The degree of spin filtering thus achieved can be quantified directly as the ratio between the spin-up and spindown transmittances at the Fermi level (38 for a single-Mo defect and about 32000 for an Mo dimer defect) or as the polarization ratio $P$, defined as the ratio between the difference and sum of these transmittances:

$$
P=\frac{T_{\uparrow}\left(E_{\mathrm{F}}\right)-T_{\downarrow}\left(E_{\mathrm{F}}\right)}{T_{\uparrow}\left(E_{\mathrm{F}}\right)+T_{\downarrow}\left(E_{\mathrm{F}}\right)} .
$$

$P$ is 0.95 for a single-Mo defect and 0.99994 when an Mo dimer is removed. Note that whereas spin filtering due to a single-atom defect would be greatly attenuated by an applied voltage greater than about $0.1 \mathrm{~V}$, which would bring the spindown transmittance peaks below the Fermi level into the energy window $\left(E_{\mathrm{F}}-0.5 \mathrm{eV}, E_{\mathrm{F}}+0.5 \mathrm{eV}\right)$, the absence of an Mo dimer makes the spin-down transmittance almost zero in a broad window around the Fermi level, thereby creating an almost perfect spin filter for a large range of applied voltages.

\section{Conclusions}

According to calculations performed using SIESTA [18] and SMEAGOL [23] for Mo-passivated $N$-ZGNRs $(N=5-10)$, in which Mo chains are bound covalently to the $\mathrm{C}$ atoms of both edges of the nanoribbon, these systems are nonmagnetic and accordingly unfit for spintronic applications. One-atomthick Mo chains adsorbed on $N$-HPZGNRs exist as chains of dimers, with Mo binding energies that are much greater when the Mo chain lies at the edge of the ribbon, particularly above edge bay sites, than at its center. The atoms of edge-bay-borne Mo chains acquire weak, ferromagnetically coupled magnetic moments while almost totally suppressing the magnetic moments of the $\mathrm{C}$ atoms of that half of the nanoribbon, Ferro-A and Ferro-F configurations of these systems differing only in that their Mo magnetic moments are respectively antiparallel and parallel to those of the $\mathrm{C}$ atoms of the opposite edge. In both Ferro-A and Ferro-F configuration, the Mo $s p_{z}$ band provides a transmission channel for each spin at the Fermi level, while the HPZGNR $\pi^{*}$ band only provides a channel for the majority spin, with the result that current through these systems is partially polarized. The Mo $s p_{z}$ channels are suppressed partially by removal of a single atom from the Mo chain and almost totally by a dimer vacancy, which creates a perfect spin filter. These findings open new prospects for the design of ZGNR-based spin-valves for integration in nanoelectronic devices. 


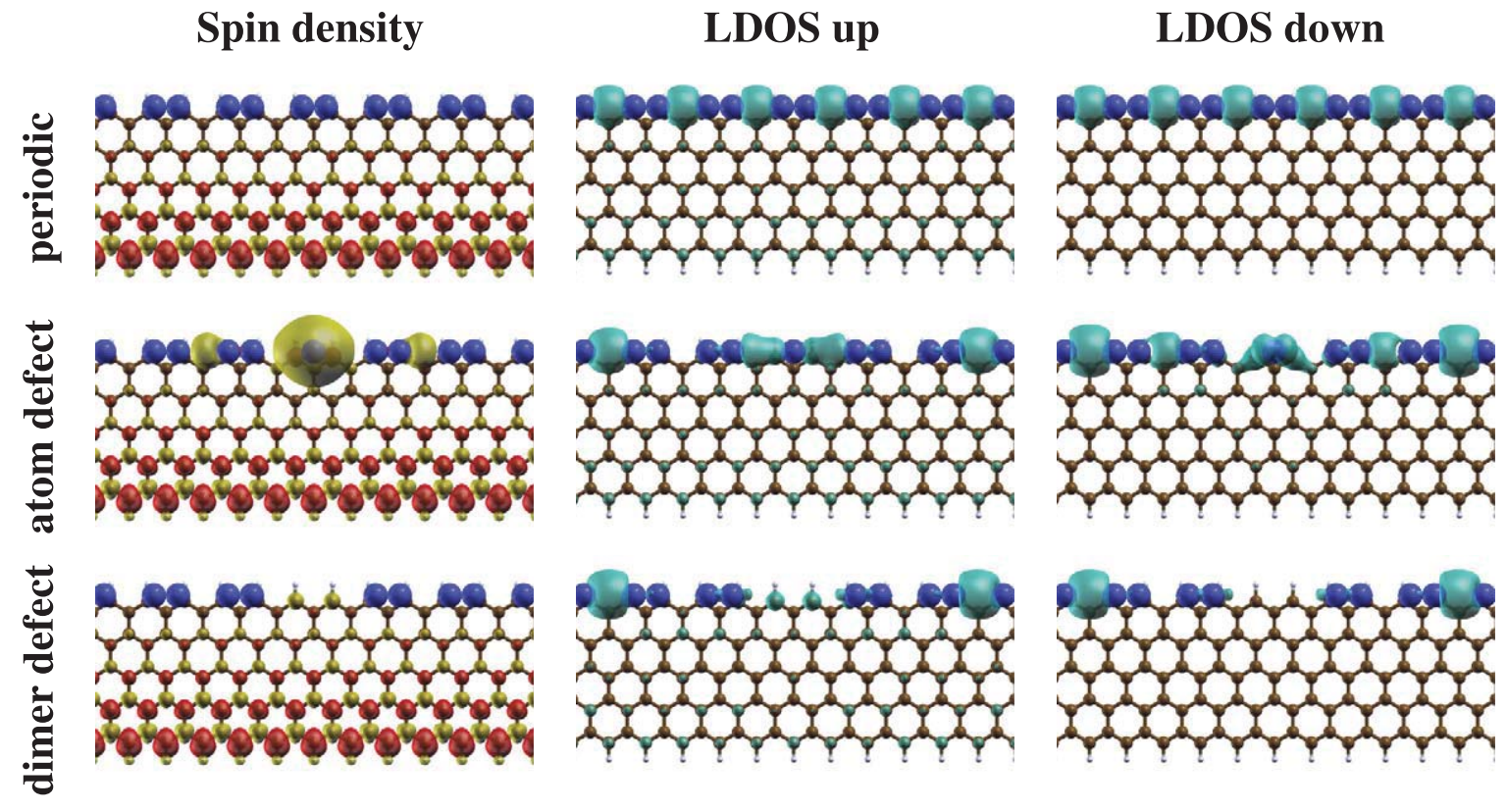

Figure 12. Spin density distribution (left) and local density of states (LDOS) around the Fermi level for each spin component (center and right) of Ferro-A Mo(a)@6-HPZGNR systems with no missing Mo atoms (top), a single-atom Mo vacancy (middle) and a missing Mo dimer (bottom). For the spin density, red (dark shading) indicates positive values (net spin up), yellow (light shading) negative values (net spin down).

\section{Acknowledgments}

We thank I-C Coleman for careful revision of the manuscript and useful suggestions. This work was supported by the Spanish Ministry of Science and Innovation (Projects FIS2011-22957 and FIS2012-33126) and by the Xunta de Galicia (Project GPC2013-043) in conjunction with the European Regional Development Fund (FEDER). AG-F acknowledges the Swiss National Science Foundation for financial support.

\section{References}

[1] Castro Neto A H, Guinea F, Peres N M R, Novoselov K S and Geim A K 2009 Rev. Mod. Phys. 81 109-62

[2] Yazyev O V 2010 Rep. Prog. Phys. 73056501

[3] Jiang D E and Chen Z 2013 Graphene Chemistry (New York: Wiley)

[4] Son Y W, Cohen M L and Louie S G 2006 Phys. Rev. Lett. 97216803

[5] Martins T B, Miwa R H, da Silva A J R and Fazzio A 2007 Phys. Rev. Lett. 98196803

[6] Pisani L, Chan J A, Montanari B and Harrison N M 2007 Phys. Rev. B 75064418

[7] Rigo V A, Martins T B, da Silva A J R, Fazzio A and Miwa R H 2009 Phys. Rev. B 79075435

[8] Son Y W, Cohen M L and Louie S G 2006 Nature 7117 347-9

[9] Martins T B, da Silva A J R, Miwa R H and Fazzio A 2008 Nano Lett. 8 2293-8
[10] Cocchi C, Prezzi D, Calzolari A and Molinari E 2010 J. Chem. Phys. 133124703

[11] Ong S V, Robles R and Khanna S N 2010 Chem. Phys. Lett. 492 127-30

[12] Wang Y, Cao C and Cheng H-P 2010 Phys. Rev. B 82205429

[13] Cao C, Wang Y, Cheng H-P and Jiang J-Z 2011 Appl. Phys. Lett. 99073110

[14] Nguyen N B, García-Fuente A, Lebon A, Gallego L J and Vega A 2012 J. Phys.: Condens. Matter 24455304

[15] Morse M D 1986 Chem. Rev. 86 1049-109

[16] Aguilera-Granja F, Vega A and Gallego L J 2008 Nanotechnology 19145704

[17] García-Fuente A, Vega A, García-Suárez V M and Ferrer J 2010 Nanotechnology 21095205

[18] Soler J M, Artacho E, Gale J D, García A, Junquera J, Ordejón P and Sánchez-Portal D 2002 J. Phys.: Condens. Matter 142745

[19] Perdew J P, Burke K and Ernzerhof M 1996 Phys. Rev. Lett. $773865-8$

[20] Troullier N and Martins J L 1991 Phys. Rev. B 43 1993-2006

[21] Kleinman L and Bylander D M 1982 Phys. Rev. Lett. 48 1425-8

[22] Press W H, Teukolsky S A, Vetterling W T and Flannery B P 2007 Numerical Recipes (Cambridge: Cambridge University Press)

[23] Rocha A R, García-Suárez V M, Bailey S, Lambert C, Ferrer J and Sanvito S 2006 Phys. Rev. B 73085414

[24] Datta S 1995 Electronic Transport in Mesoscopic Systems (Cambridge: Cambridge University Press)

[25] García-Fuente A, García-Suárez V M, Ferrer J and Vega A 2011 J. Phys.: Condens. Matter 23265302 